\title{
Photooxidation and Photobleaching of Single CdSe/ZnS Quantum Dots Probed by Room-Temperature Time-Resolved Spectroscopy
}

\author{
Wilfried G. J. H. M. van Sark, ${ }^{* \dagger}$ Patrick L. T. M. Frederix, \\ Hans C. Gerritsen \\ Department of Molecular Biophysics, Debye Institute, Utrecht University, P.O. Box 80000, \\ 3508 TA Utrecht, The Netherlands \\ Ageeth A. Bol, Joost N. J. van Lingen, Celso de Mello Donegá, and Andries Meijerink \\ Department of Physics and Chemistry of Condensed Matter, Debye Institute, Utrecht University, \\ P.O. Box 80000, 3508 TA Utrecht, The Netherlands
}

Received: May 24, 2001; In Final Form: July 16, 2001

\begin{abstract}
The room-temperature luminescence of single $\mathrm{CdSe} / \mathrm{ZnS}$ core-shell quantum dots is investigated by spectrally and temporally resolved confocal microscopy. A large $(30 \mathrm{~nm})$ blue shift is observed in the emission wavelength during illumination in air. In nitrogen, no blue shift is observed. The blue shift in air is ascribed to a $1 \mathrm{~nm}$ shrinkage of the CdSe core by photooxidation. Photobleaching occurs about 4 times faster in air than in nitrogen, indicating the formation of nonradiative recombination centers during photooxidation. The initial light output is higher in air than in nitrogen, which may be due to a reduction of the defect state lifetime by oxygen.
\end{abstract}

\section{Introduction}

One of the unique properties of semiconductor quantum dots is the change in the electronic structure as a function of particle size. ${ }^{1-3}$ Because of the confinement of electrons and holes in the nanometer sized crystallites the energy level scheme resembles that of an atom, with many discrete energy levels. The separation between energy levels increases as the particle size decreases. It has been recently demonstrated that it is possible to measure emission spectra for a single quantum dot., ${ }^{4,5}$ Since then, the study of the luminescence of single semiconductor quantum dots has revealed many interesting properties that cannot be observed for an ensemble of many quantum dots. Phenomena of fundamental interest like spectral diffusion and blinking (on/off behavior) are also important for potential applications of single quantum dots as luminescent labels in biological systems. ${ }^{6,7}$ Most studies on spectral diffusion have been performed at cryogenic temperatures. Random spectral diffusion at these low temperatures has been related to ionization of quantum dots due to Auger processes..$^{5,8-11}$ As a result of the ionization and subsequent recombination processes, the charge distribution around the dot changes, resulting in a spectral (Stark) shift of the emission. ${ }^{11,12}$ Convincing evidence has been presented for this mechanism. ${ }^{11}$ In addition, a clear relation between blinking (explained by photoionization) and the occurrence of a spectral jump has been established.

At room-temperature, less information is available on spectral diffusion of single QD luminescence. The spectral diffusion at room temperature is not random, but rather, a blue shift of about $10-15 \mathrm{~nm}$ has been reported. ${ }^{5,13}$ This blue shift was attributed

* To whom correspondence should be addressed. Tel: +31302532825. Fax: +31 30253 2706. E-mail: W.G.J.H.M.vanSark@phys.uu.nl.

$\dagger$ W. G. J. H. M. van Sark and P. L. T. M. Frederix contributed equally to this research.

$\doteqdot$ Also at Department of Medical Physiology, Faculty of Medicine, Utrecht University, P.O. Box 80030, 3508 TA Utrecht, The Netherlands. to irreversible photooxidation of the quantum dot. In contrast, also photoinduced surface transformation has been reported which recovers fluorescence quantum yield. ${ }^{14}$

In this letter, we investigate the time evolution of the roomtemperature emission spectra of single $\mathrm{CdSe} / \mathrm{ZnS}$ quantum dots over time periods up to $30 \mathrm{~min}$ with a time resolution down to $6 \mathrm{~ms}$. The spectral diffusion (blueing), the on/off behavior (blinking), and the disappearance of the emission (bleaching) are studied for two types of CdSe quantum dots (varying in the thickness of the $\mathrm{ZnS}$ shell) both in air and in nitrogen.

\section{Materials and Methods}

The ZnS-capped CdSe nanoparticles were synthesized by using a TOP/TOPO method similar to the one described by Hines and Guyot-Sionnest. ${ }^{15}$ Two batches of overcoated dots were synthesized. For batch 1, about 4 monolayers of $\mathrm{ZnS}$ were grown over the CdSe core, whereas for batch 2, the thickness amounted to about 7 monolayers. The nanocrystals were precipitated by adding anhydrous methanol and, after centrifuging, dispersed in doubly distilled chloroform. The average emission wavelength was about $600 \mathrm{~nm}$, which corresponds to an average diameter of the CdSe core of quantum dots from both batches of about $5 \mathrm{~nm} .{ }^{1}$ The polydispersity in the two batches was about $50 \%$, as was deduced from the rather broad emission spectrum of the ensemble average of nanocrystals. The luminescence quantum efficiency at room temperature was determined to be about $30 \%$. For single particle luminescence measurements, small droplets of the strongly diluted QD stock solution were deposited, spread out, and dried on cover glass slides. The final density was approximately $0.1 \mathrm{dot} / \mu \mathrm{m}^{2}$. The slides were prepared, mounted, and sealed in a flow chamber in nitrogen. Prior to and during the experiments, nitrogen or air was flushed through the flowchamber.

Fast spectral imaging of single dots was performed employing a confocal laser scanning microscope (CLSM, Nikon PCM2000). 


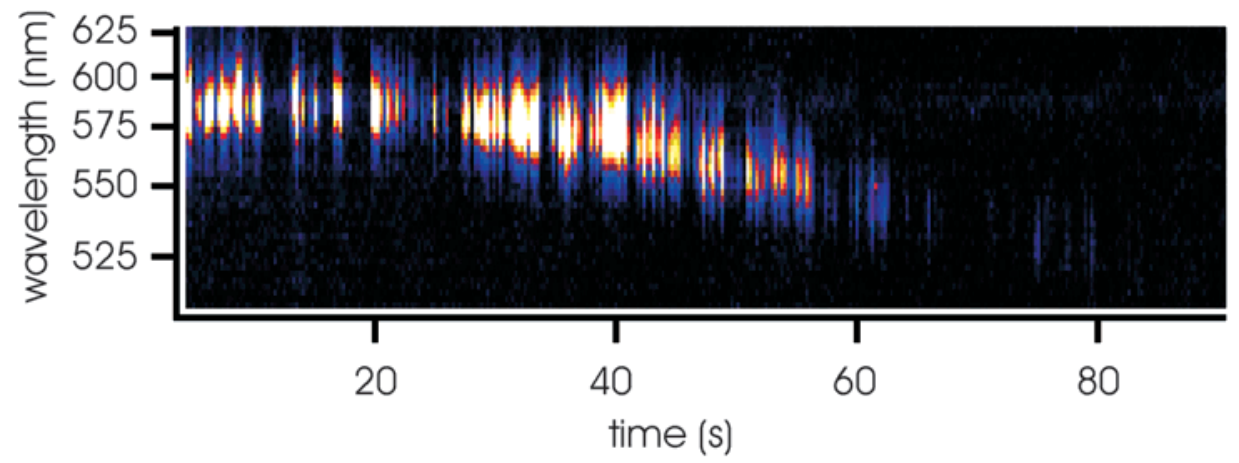

(a)

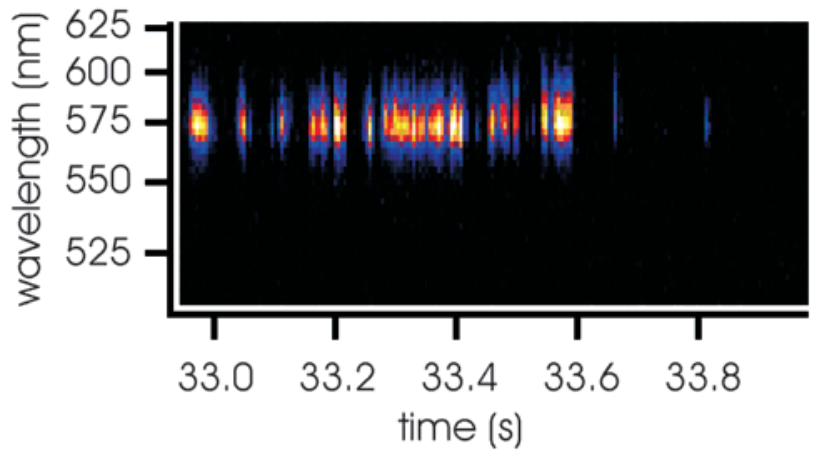

(b)

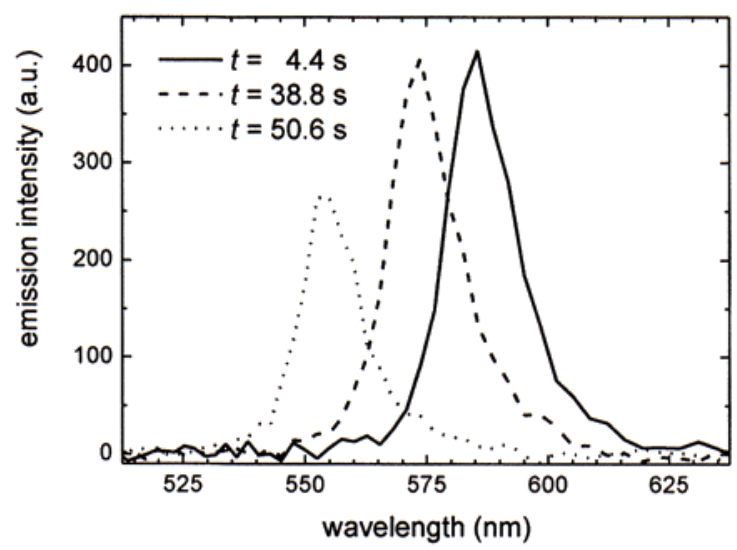

(c)

Figure 1. Spectrally resolved time trace (a) of a CdSe/ZnS QD of batch 1 in ambient air present in the detection volume of the CLSM/spectrograph (excitation at $468 \mathrm{~nm}$, room temperature). Note that the intensity is a stretched false color representation. The blow-up (b) shows a smaller part of the time trace. Emission spectra at different illumination times are shown in c.

The $468 \mathrm{~nm}$ line of an $\mathrm{Ar}-\mathrm{Kr} \mathrm{CW}$ laser was used for excitation (power $\approx 20 \mathrm{~kW} / \mathrm{cm}^{2}$ ). The detection channels of the microscope were coupled by optical fibers to the detectors. One of the two standard detectors was replaced by a home-built spectrograph. ${ }^{16}$ In the spectrograph, the light was dispersed by a prism, and detected with a Peltier-cooled, back-illuminated CCD camera (Princeton Instruments, NTE/CCD-1340). The spectral resolution varied between 1 and $7 \mathrm{~nm}$, in the wavelength range from 450 to $750 \mathrm{~nm}$. In the measurements presented here, the emission was collected using a $60 \times / 1.4$ oil immersion objective (NIKON, PlanApo). The photomultiplier of the CLSM was first used to locate a single QD, applying a 590/60 band-pass filter. The laser beam was parked at the position of single QDs, and spectra were recorded with a $6 \mathrm{~ms}$ dwell time. The luminescence of QDs was followed until the luminescence had been bleached away.

\section{Results and Discussion}

Luminescence spectra of individual $\mathrm{CdSe} / \mathrm{ZnS}$ quantum dots were measured for a large number of quantum dots. Clear differences are observed between the time evolution of the emission spectra of the quantum dots, even for dots of the same batch in the same atmosphere. To obtain reliable information on the influence of the atmosphere and the thickness of the capping layer on the spectral diffusion and on/off behavior, the emission spectra of 41 different dots were followed in time. In Figure 1a a typical spectrally resolved time trace (duration 80 s) is presented of a $\mathrm{CdSe} / \mathrm{ZnS} \mathrm{QD}$ of batch 1 in air. Initially this QD emits at $585 \mathrm{~nm}$ (for about $20 \mathrm{~s}$ ), and then the emission wavelength starts to shift to the blue. After a blue shift of about $40 \mathrm{~nm}$, the emission of the QD is fully photobleached. A blowup (Figure $1 \mathrm{~b}$ ) of a smaller part of the time trace shows blinking of the QD. Figure 1c shows spectra of the dot collected $(6 \mathrm{~ms}$ integration) at different times. The two spectra recorded at the later times are clearly blue shifted with respect to the initial wavelength. The position of the maxima, the integrated intensity, and the full width at half-maximum (fwhm) of the emission bands were determined by fitting the spectra with a Lorentzian. ${ }^{3}$ The fwhm of the peaks is about $15 \mathrm{~nm}$. This line width is in agreement with the homogeneous broadening by phonon dephasing processes. ${ }^{17}$ The result of fitting of the emission maxima is shown in Figure 2, where the emission wavelength of a CdSe/ZnS QD from batch 1 in air is depicted as a function of time. Similar results are obtained for other quantum dots of batchs 1 and 2 in air, with a blue shift of $29 \pm 10 \mathrm{~nm}$ and 29 $\pm 17 \mathrm{~nm}$, respectively, before the luminescence disappears. In 


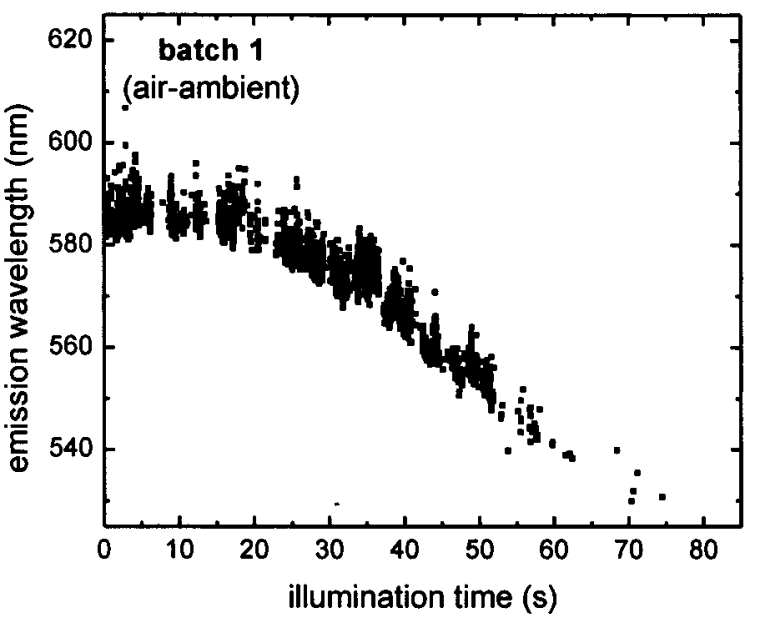

Figure 2. Emission wavelength as a function of time for the CdSe/ $\mathrm{ZnS}$ QD of Figure 1.

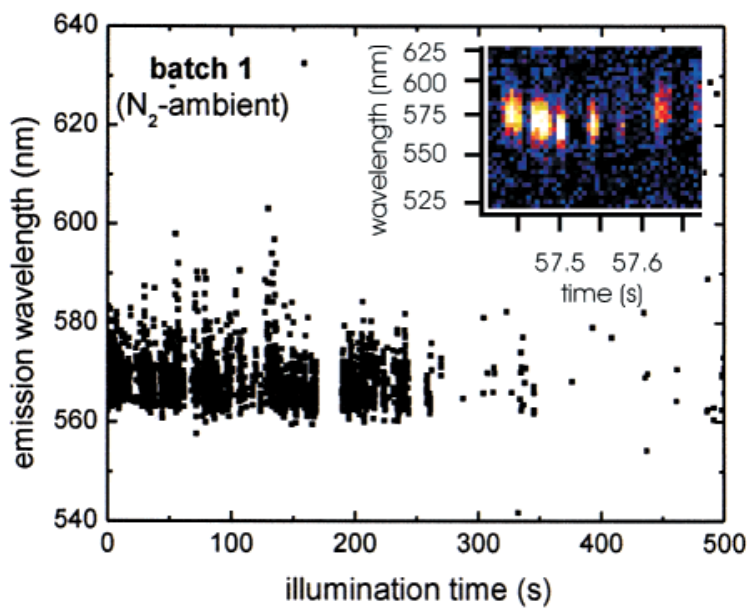

Figure 3. Emission wavelength as a function of time for a $\mathrm{CdSe} / \mathrm{ZnS}$ QD of batch 1 in a nitrogen atmosphere. The inset shows a spectrally resolved time trace revealing the spectral jumps.

some cases, an initial fast blue shift is observed, whereas for most QDs, a behavior similar to that depicted in Figure 2 is observed. For QDs of batch 1, the luminescence intensity decreases in time and no luminescence is observed after typically $2.5 \mathrm{~min}$. There are large differences between quenching times for individual dots, in agreement with previously reported results. ${ }^{5}$ Because of the increased thickness of the passivating $\mathrm{ZnS}$ layer for QDs from batch 2, the time scales for the blue shift and the photobleaching are significantly longer (bleaching occurs on average after $3.5 \mathrm{~min}$ ).

The time-evolution of the emission wavelength for a single $\mathrm{CdSe} / \mathrm{ZnS}$ QD of batch 1 in nitrogen atmosphere is depicted in Figure 3. Clearly, no blue shift is observed. The emission wavelength of this QD varies around an average value of about $570 \mathrm{~nm}$ (Figure 3). A spectral variation of $10 \mathrm{~nm}$ is observed. This is in agreement with previous measurements, which show that over longer time periods random spectral diffusion can result in shifts of up to $10 \mathrm{~nm} .^{18,19}$ This spectral diffusion is further illustrated in the inset of Figure 3, where clear jumps in the emission wavelength are visible. Contrary to the situation in air, there is only random spectral diffusion in time and no shift to shorter wavelengths is observed. The time periods for which the dots show emission (until photobleaching occurs) are significantly longer for the QDs in nitrogen than in air (about $10 \mathrm{~min}$ on average for both batches).
The presently observed differences between the time evolution of the emission spectra of single quantum dots in air and in nitrogen provide convincing evidence that the observed blue shift of the emission in air is due to photooxidation of CdSe. From the blue shift and the well-known relation between band gap and the diameter of CdSe particles, ${ }^{1-3}$ it can be calculated that the effective CdSe core diameter decreases from about 5 $\mathrm{nm}$ to about $4 \mathrm{~nm}$ before the dot is completely bleached. A change in particle diameter of $1 \mathrm{~nm}$ corresponds to photooxidation of almost two layers of CdSe from the surface. ${ }^{5}$ Oxidation of CdSe nanocrystals is well-known. Even under ambient conditions (without intense excitation), surface oxidation of $\mathrm{CdSe}$ nanoparticles has been reported. ${ }^{20}$ The main oxidation product was suggested to be $\mathrm{SeO}_{2}$. Photooxidation is well understood for CdS nanocrystals. ${ }^{21-23}$ Upon illumination, CdS nanocrystals in solution are photooxidized to $\mathrm{Cd}^{2+}$ and $\mathrm{SO}_{4}{ }^{2-}$. For photooxidation of $\mathrm{CdSe}$, evidence for the formation of $\mathrm{CdSeO}_{x}$ has been found, i.e., $x=2^{1,20}$ and $x=3 .^{21}$ In the present experiments, the nature of the photooxidation product has not been analyzed. For photooxidation to take place, oxygen has to diffuse through the passivating $\mathrm{ZnS}$ layer that has been grown on the CdSe nanocrystals. The observation that photooxidation occurs indicates that the $\mathrm{ZnS}$ layer is not a closed epitaxial layer but rather a layer with grain boundaries, presumably at places where $\mathrm{ZnS}$ islands, which started to grow at different locations on the CdSe nanocrystal, meet. At these boundaries, oxygen can diffuse to the $\mathrm{CdSe}$ core inside the $\mathrm{ZnS}$ shell. For the thicker shell (batch 2) the oxidation rate is reduced because of the slower diffusion of oxygen to the $\mathrm{CdSe}$ core through a thicker $\mathrm{ZnS}$ shell.

As a result of the photooxidation at the CdSe surface, quenching states are expected to be formed at the $\mathrm{CdSe} / \mathrm{CdSeO}_{x}$ interface. The formation of surface quenching states causes a decrease of the number of photons emitted. In the single QD emission spectra, a gradual decrease in light output is indeed observed as the emission shifts to shorter wavelengths. Finally, the luminescence disappears and the dot has bleached. The occurrence of photooxidation for QDs can explain the shorter bleaching times observed for QDs in air. Also, in a nitrogen atmosphere, photobleaching occurs, albeit after much longer times. In view of the high laser power $\left(20 \mathrm{~kW} / \mathrm{cm}^{2}\right)$, photobleaching is not unexpected. Few materials are stable against photodegradation under the presently used laser power. The nature of the photoinduced quenching states is not clear. The efficiency of the photoinduced formation of quenching states in nitrogen is much lower than for photooxidation observed in air. Possibly, a high energy biexciton state in a single dot has enough energy to rearrange or break bonds at the $\mathrm{CdSe} / \mathrm{ZnS}$ interface which gives rise to nonradiative recombination channels and finally leads to bleaching.

The blinking behavior of the $\mathrm{ZnS}$ coated CdSe QDs is evident from Figures 1-3. Analysis of the off-time intervals shows that the probability density of off-time intervals can be described by an inverse power law, i.e., $P\left(t_{\text {off }}\right) \propto t_{\text {off }}{ }^{-(1+\alpha)}$, in accordance with Kuno et al. ${ }^{10}$ They reported an excellent agreement between the experimentally observed off-time distribution over nine decades in probability density and five decades in time, using $(1+\alpha)=1.6 \pm 0.1$. For our 41 dots, we find an average value of $(1+\alpha)=1.4 \pm 0.2$, in correspondence to earlier reported values. ${ }^{10,24}$ The most probable explanation for the inverse power law behavior is the existence of multiple ionization states and consequently a distribution of recombination rates. ${ }^{10}$ Because the process that leads to the off state is assigned to photoionization of the $\mathrm{QD}$, the off-time intervals are related to 
recombination of the ejected charge carrier with the ionized dot. ${ }^{10}$ Therefore, a difference in blinking behavior in air and in nitrogen is expected as the shrinking of the CdSe core and the formation of $\mathrm{CdSeO}_{x}$ at the interface is likely to influence the tunneling rate for recombination of an ejected charge carrier and an ionized dot. Preliminary results seem to indicate that somewhat lower values of $(1+\alpha)$ are obtained for QDs in nitrogen in comparison to oxygen, i.e., average off-times in nitrogen are somewhat longer. From a recent study by Koberling et al., ${ }^{25}$ a small difference in off-time distribution can be inferred between blinking in argon and in oxygen. At present, further research is in progress to study in detail differences in the blinking of QDs in air and nitrogen.

The potential application of single quantum dots as luminescent labels (e.g., in biological systems) is based on the high stability in combination with a relative narrow emission band, a broad excitation band, and a large "Stokes shift". ${ }^{6,7}$ The total number of photons emitted by a single QD until bleaching occurs is an important number. For single dye molecules (for example for rhodamine ${ }^{26-28}$ ), the highest number of photons emitted before bleaching is around $10^{6}$ at room temperature. In the various papers on the luminescence of single QDs, we have not found an estimate for the number of photons emitted by a single QD. On the basis of an overall efficiency of about 5\% for the system (i.e., one out of every twenty emitted photons is counted), the number of photons emitted by a single QD before bleaching occurs is typically $2 \times 10^{7}$, with numbers exceeding $10^{8}$ for the more robust quantum dots. These numbers are more than an order of magnitude higher than those for single dye molecules, which reflects the higher stability of inorganic chromophores such as semiconductor QDs.

Surprisingly, the total number of photons emitted before bleaching occurs is not much less for QDs in air than for QDs in nitrogen. The shorter lifetime of QDs in air is compensated for by a higher initial photon count in air. The initial emission intensity is about two times higher for QDs in air (about 3000 counts in air vs 1500 counts in nitrogen during the $6 \mathrm{~ms}$ integration period for the brightest QDs). A possible explanation for the higher initial light output in air is quenching of QD defect luminescence by oxygen. It is well-known that in addition to the fast (nanosecond) exciton emission, also relatively longlived (microsecond) defect emission can occur in QDs. ${ }^{21,29}$ Because of the long lifetime, the fast photon absorption and emission process is interrupted, until the long-lived excited state has returned (via radiative or nonradiative relaxation) to the ground state. If oxygen can quench the defect luminescence, a higher exciton emission yield is expected by reduction of the time spent in the "dark state". For CdS and $\mathrm{ZnS}$, it has been established that oxygen can quench the defect related emission. ${ }^{21,29}$ If the same occurs for CdSe QDs, this can explain the higher initial light output observed for CdSe QDs in air. The presently applied excitation power $\left(20 \mathrm{~kW} / \mathrm{cm}^{2}\right)$ yields initial intensities of 1000-3000 counts in $6 \mathrm{~ms}$. Assuming a 5\% collection efficiency, this corresponds to time intervals of 100$300 \mathrm{~ns}$ between emission of photons. In this situation, relaxation to a trap state with a microsecond lifetime will reduce the number of photons emitted and a fast return to the ground state by nonradiative relaxation enhances the photon output. A similar situation has been reported for dye molecules where higher fluorescence light yields were measured by quenching of the triplet state luminescence. ${ }^{30}$ Very recently, it has been reported that probably the adsorption of water molecules in stead of oxygen is responsible for the strong influence on fluorescence intensity. ${ }^{13,31}$

\section{Conclusion}

In this letter, we have presented time-resolved luminescence measurements on single $\mathrm{CdSe} / \mathrm{ZnS}$ core-shell QDs. A clear blue shift in the emission wavelength of $30 \mathrm{~nm}$ is observed for QDs in ambient air. In contrast, this shift is not observed for QDs in a nitrogen atmosphere. Moreover, the bleaching time and initial emission intensity of single QDs are influenced by the presence of oxygen. The results are explained by photoinduced oxidation of the CdSe crystallites.

Acknowledgment. We gratefully acknowledge The Netherlands Technology Foundation (STW) and The Netherlands Council for Earth and Life Sciences (ALW) of The Netherlands Organization for Scientific Research (NWO) for financial support.

\section{References and Notes}

(1) Dabbousi, B. O.; Rodriguez-Viejo, J.; Mikulec, F. V.; Heine, J. R.; Mattoussi, H.; Ober, R.; Jensen, K. F.; Bawendi, M. G. J. Phys. Chem. B 1997, 101, 9463 .

(2) Empedocles, S.; Bawendi, M. G. Acc. Chem. Res. 1999, 32, 389.

(3) Gaponenko, S. V. Optical Properties of Semiconductor Nanocrystals; Cambridge University Press: Cambridge, U.K., 1998.

(4) Blanton, S. A.; Dehestani, A.; Lin, P. C.; Guyot-Sionnest, P. Chem. Phys. Lett. 1994, 229, 317.

(5) Nirmal, M.; Dabbousi, B. O.; Bawendi, M.; Macklin, J. J.; Trautmann, J. K.; Harris, T. D.; Brus, L. E. Nature 1996, 383, 802.

(6) Bruchez, M., Jr.; Moronne, M.; Gin, P.; Weiss, S.; Alivisatos, A. P. Science 1998, 281, 2013.

(7) Chan, W. C. W.; Nie, S. Science 1998, 281, 2016.

(8) Efros, Al. L.; Rosen, M. Phys. Rev. Lett. 1997, 78, 1110.

(9) Banin, U.; Bruchez, M.; Alivisatos, A. P.; Ha, T.; Weiss, S.; Chemla, D. S. J. Chem. Phys. 1999, 110, 1195

(10) Kuno, M.; Fromm, D. P.; Hamann, H. F.; Gallagher, A.; Nesbitt, D. J. J. Chem. Phys. 2000, 112, 3117.

(11) Neuhauser, R. G.; Shimizu, K. T.; Woo, W. K.; Empedocles, S. A.; Bawendi, M. G. Phys. Rev. Lett. 2000, 85, 3301.

(12) Empedocles, S. A.; Bawendi, M. G. Science 1997, 278, 2114.

(13) Cordero, S. R.; Carson, P. J.; Estabrook, R. A.; Strouse, G. F.; Buratto, S. K. J. Phys. Chem. B 2000, 104, 12137.

(14) Hess, B. C.; Okhrimenko, I. G.; Davis, R. C.; Stevens, B. C.; Schulzke, Q.; Wright, K. C.; Bass, C. D.; Evans, C. D.; Summers, S. L. Phys. Rev. Lett. 2001, 86, 3132.

(15) Hines, M. A.; Guyot-Sionnest, P. J. Phys. Chem. 1996, 100, 468.

(16) Frederix, P. L. T. M.; Asselbergs, M. A. H.; Van Sark, W. G. J. H. M.; Van den Heuvel, D. J.; Hamelink, W.; De Beer, E. L.; Gerritsen, H. C Appl. Spec. 2001, 55, in press.

(17) Li, X.-Q.; Arakawa, Y. Phys. Rev. B 1999, 60, 1915.

(18) Blanton, S. A.; Hines, M. A.; Guyot-Sionnest, P. Appl. Phys. Lett. 1996, 69, 3905.

(19) Empedocles, S. A.; Neuhauser, R.; Shimizu, K.; Bawendi, M. G. Adv. Mater. 1999, 11, 1243.

(20) Bowen Katari, J. E.; Colvin, V. L.; Alivisatos, A. P. J. Phys. Chem. 1994, 98, 4109.

(21) Henglein, A. Top. Curr. Chem. 1988, 143, 113.

(22) Dunstan, D. E.; Hagfeldt, A.; Almgren, M.; Siegbahn, H. O. G.; Mukhtar, E. J. Phys. Chem. 1990, 94, 6797.

(23) Spanhel, L.; Haase, M.; Weller, H.; Henglein, A. J. Am. Chem. Soc. 1987, 109, 5649 .

(24) Van Sark, W. G. J. H. M.; Frederix, P. L. T. M.; Van den Heuvel, D. J.; Asselbergs, M. A. H.; Senf, I.; Gerritsen, H. C. Single Mol. 2000, 1, 291.

(25) Koberling, F.; Mews, A.; Basché, T. Adv. Mater. 2001, 13, 672.

(26) Rosenthal, I. Opt. Comm. 1978, 24, 164.

(27) Huston, A. L.; Reimann, C. T. Chem. Phys. 1991, 149, 401.

(28) Schmidt, Th.; Schütz, G. J.; Baumgartner, W.; Gruber, H. J.; Schindler, H. Proc. Natl. Acad. Sci. U.S.A. 1996, 93, 2926.

(29) Chestnoy, N.; Harris, T. D.; Hull, R.; Brus, L. E. J. Phys. Chem. 1986, 90, 3393

(30) Song, L.; Varma, C. A. G. O.; Verhoeven, J. W.; Tanke, H. J. Biophys. J. 1996, 70, 2959.

(31) Ladizhansky, V.; Hodes, G.; Vega, S. J. Phys. Chem. B 2000, 104, 1939. 